ISSN 2766-5879

\title{
A Summary Note of Gestational Diabetes Control Centred on Several Evidence-Based Type 2 Diabetes Research Results Using GH-Method: Math-Physical Medicine (No. 399)
}

Gynecology and Women's Health Care

Research Article

\section{Gerald C Hsu}

eclaireMD Foundation, USA

\author{
*Correspondence author \\ Gerald C Hsu \\ eclaireMD Foundation \\ USA
}

Submitted : 9 Mar 2020 ; Published : 22 Mar 2021

\begin{abstract}
The author, who has type 2 diabetes (T2D) for 26 years, conducted his diabetes research over the past 11 years. Since 2020, he has published 400+ medical research papers in various medical journals.

Recently, he received invitations to submit his medical research notes to a gynecology journal but he hesitated because he was not a gynecologist. However, the editor convinced him that his research results may be beneficial to some patients with gestational diabetes mellitus (GDM) since diabetes conditions are quite similar, if not the same. Therefore, he started to review some medical papers online on the subject of gestational diabetes. Finally, he uncovered that his research results could indeed benefit some GDM patients to some degree and decided to write this summary note.
\end{abstract}

Although his research specialties are in the areas of endocrinology, diabetes, and lifestyle, after 11 years of dedicated medical research with additional self-studying on GDM, he discovered that approximately $90 \%$ of his diabetes research findings are applicable to this special group.

In summary, three areas with special emphasis are highlighted for GDM patients.

The first and major area is food nutrition in a lifestyle management program. Due to the concerns of hyperglycemia, the author eats high-quality protein, a lot of fish and vegetables, particularly green leafy vegetables, portioncontrolled shellfish and fruits, along with avoidance of red meat and sweetened food. However, for a GDM patient, he highly recommends them to follow a "nutritional balanced" meal plan, avoiding high-carbs, sugar-based, and processed food in general to prevent unhealthy chemical additives. The fetus in utero needs good nutrition supplied by its mother; therefore, the mother must eat nutritional balanced meals that meet nutritional requirements and glucose concerns.

The second area is specific weight-gain management. The author focuses on the food portion percentage with a strong willpower to resist his food cravings plus persistence each day, so that he can achieve his weight reduction target. However, for a GDM patient, she must constantly monitor the amount of weight gained according to the Mayo Clinic's "weight-gain guidelines". Maintenance in this area is important for the benefit of the baby's development and mother's health during pregnancy. However, both of his food portion percentage and persistent weight control are good examples to follow and useful for a GDM patient.

The third area is medication treatment for gestational diabetes. Although the author ceased taking medications in controlling his diabetes symptoms, he decided to implement a rather stringent lifestyle management program in order to control or even reverse his diabetes conditions at the root-cause level. Nevertheless, it is a difficult route that takes a longer time span to be able to see significant improvements. Since the pregnancy period is relatively shorter, less than 10 months, a gestational diabetes patient must follow her physician's advice to pursue all the necessary medication treatments or insulin injections.

The information in particular the high correlation coefficients between any two variables, from Figures 2 through 5, provide useful knowledge for a GDM patient to learn in order to control her glucose levels during pregnancy. The most important goal is the safe delivery of a healthy new born. 


\section{Introduction}

The author, who has type 2 diabetes (T2D) for 26 years, conducted his diabetes research over the past 11 years. Since 2020, he has published $400+$ medical research papers in various medical journals.

Recently, he received invitations to submit his medical research notes to a gynecology journal but he hesitated because he was not a gynecologist. However, the editor convinced him that his research results may be beneficial to some patients with gestational diabetes mellitus (GDM) since diabetes conditions are quite similar, if not the same. Therefore, he started to review some medical papers online on the subject of gestational diabetes. Finally, he uncovered that his research results could indeed benefit some GDM patients to some degree and decided to write this summary note.

\section{Methods}

\section{GH-Method: MPM Background}

To learn more about his developed GH-Method: math-physical medicine (MPM) methodology, readers can read the following three papers selected from the published $400+$ medical papers.

The first paper, No.386 (Reference 1) describes his MPM methodology in a general conceptual format. The second paper, No.387 (Reference 2) outlines the history of his personalized diabetes research, various application tools, and the differences between biochemical medicine (BCM) approach versus the MPM approach. The third paper, No.397 (Reference 3) depicts a general flow diagram containing $\sim 10$ key MPM research methods and different tools.

\section{Other Gestational Diabetes articles}

There are many available articles on GDM studies; however, the author has read several published papers on this subject and decided to combine some of their key points into one excerpt (References 4 through 11). This allows the readers to get a quick overview.

\section{Here is the excerpt}

"Gestational diabetes mellitus (GDM) is defined as any degree of glucose intolerance first detected during pregnancy regardless of the degree of hyperglycemia although this definition has serious limitations. The age of a mother, prepregnancy body mass index (BMI), the amount of obesity, family history, and weight gain during pregnancy are GDMrelated factors.

It is estimated that GDM affects around 7\% to $10 \%$ of all pregnancies worldwide, resulting in more than 200,000 cases annually. Nearly $10 \%$ of pregnancies in the U.S. are affected by gestational diabetes every year. However, comparing GDM prevalence between countries has been confounded by differing sets of diagnostic criteria. The absence of universal gold standards for screening of GDM has led to heterogeneity in the identification of GDM, thereby impacting the accurate estimation of the prevalence of GDM, related health outcomes as well as their health costs. The prevalence may range from 1 to $14 \%$ of all pregnancies, depending on the population studied and the diagnostic tests employed.

Diabetes confers significantly greater maternal and fetal risk largely related to the degree of hyperglycemia but also related to chronic complications and comorbidities of diabetes. In general, specific risks of diabetes in pregnancy include spontaneous abortion, fetal anomalies, preeclampsia, fetal demise, macrosomia, neonatal hypoglycemia, hyperbilirubinemia, and neonatal respiratory distress syndrome, among others. In addition, diabetes in pregnancy may increase the risk of obesity, hypertension, and type 2 diabetes in offspring later in life.

We do know that the placenta supports the baby as it grows. Sometimes, these hormones also block the action of the mother's insulin to her body and it causes a problem called insulin resistance (IR). This insulin resistance makes it hard for the mother's body to use insulin. And this means that she may need up to three times as much insulin to compensate. Gestational diabetes can also start when the mother's body is not able to make and use all the insulin it needs for pregnancy. Without enough insulin, glucose can't leave the blood and be changed into energy. When glucose builds up in the blood, it's called "hyperglycemia".

Researchers are still trying to learn more about the link between type 2 diabetes and certain cancers, including liver, pancreas, uterus, colon, breast, and bladder. Type 2 diabetes and certain cancers share some risk factors. The good news is that some of these risk factors are within patient's control to manage. These risk factors include age, gender, race/ethnicity, overweight, inactivity, smoking, and alcohol.

Because gestational diabetes can hurt both mother and baby, it is critical to start treatment quickly. Treatment for gestational diabetes aims to keep blood glucose levels equal to those of pregnant women who don't have gestational diabetes. The treatment always includes special meal plans and scheduled physical activity, and it may also include daily glucose testing and insulin injections.

Gestational diabetes usually goes away after pregnancy. But once you've had gestational diabetes, your chances are two in three that it will return in future pregnancies. In a few women, however, pregnancy uncovers type 1 or type 2 diabetes. It is hard to tell whether these women have gestational diabetes or have just started showing their diabetes during pregnancy, but they will need to continue diabetes treatment after pregnancy. Many women who have gestational diabetes go on to develop type 2 diabetes years later. There seems to be a link between the tendency to have gestational diabetes and type 2 diabetes since both involve insulin resistance. However, certain basic lifestyle changes may help prevent diabetes after gestational diabetes. 
The ADA-recommended targets for women with type 1 or type 2 diabetes are as follows:

- Fasting glucose $<95 \mathbf{m g} / \mathbf{d L}(5.3 \mathrm{mmol} / \mathrm{L})$ and either

- One-hour postprandial glucose $<140 \mathrm{mg} / \mathrm{dL} \quad(7.8$ $\mathrm{mmol} / \mathrm{L}$ ) or

- Two-hour postprandial glucose $<120 \mathrm{mg} / \mathrm{dL}(6.7 \mathrm{mmol} / \mathrm{L})$

\section{Always remember that GDM is treatable}

Researchers don't yet know why some women get gestational diabetes and others don't. Excess weight before pregnancy often plays a role. Normally, various hormones work to keep your blood sugar levels in check. But during pregnancy, hormone levels change, making it harder for your body to process blood sugar efficiently. This makes your blood sugar rise. For most women, gestational diabetes doesn't cause noticeable signs or symptoms. Increased thirst and morefrequent urination are possible symptoms.

Women of average risk should have testing undertaken at 2428 weeks of gestation. Low-risk status requires no glucose testing, but this category is limited to those women meeting all of the following characteristics:

- $\quad$ Age $<25$ years

- Weight normal before pregnancy

- Member of an ethnic group with a low prevalence of GDM

- No known diabetes in first-degree relatives

- No history of abnormal glucose tolerance

- No history of poor obstetric outcome

A fasting plasma glucose level $>126 \mathrm{mg} / \mathrm{dl}(7.0 \mathrm{mmol} / \mathrm{l})$ or a casual plasma glucose $>200 \mathrm{mg} / \mathrm{dl}$ (11.1 mmol/l) meets the threshold for the diagnosis of diabetes. A body mass index (BMI) of 25 or greater plus an additional risk factor (e.g., physical inactivity, a first-degree relative with diabetes, highrisk ethnicity, previous GDM, hypertension) warrants early screening.

In 2014, the U.S. Preventive Services Task Force updated its 2008 statement to recommend that asymptomatic pregnant women be screened for GDM after 24 weeks of gestation. Most clinicians in the United States use a two-step approach, first administering a 50-g non-fasting oral glucose challenge test at 24 to 28 weeks, followed by a 100 -g fasting test for women who have a positive screening result. Alternatively, clinicians may use a one-step approach and administer only a 75-g twohour fasting oral glucose tolerance test. Gestational diabetes usually arises in the second half of pregnancy and goes away as soon as the baby is born. However, if gestational diabetes is not treated, you may experience complications.

Following possible complications affecting the baby include
Following possible complications affecting the mother include

- $\quad$ High blood pressure and preeclampsia

- Having a surgical delivery (C-section)

- $\quad$ Future diabetes

Studies showed that, in women with GDM, improvements on Quality of Life (QoL) can be achieved through different intervention programs by enhancing positive diabetes-related self-management behaviours. Based on current research studies, we have found that QoL could be significantly compromised, both short term and long term, when women cope with pregnancy complicated by GDM. A relatively recent review study of Lawrence has further underlined how perceptions and expectations of women with GDM may significantly affect their psychological and behavioural response during and after pregnancy.

Further research should be conducted to test the effect of integrative programs on QoL focusing on pharmacological care mixed up with advanced practices based on information and communication technologies (e.g., telemedicine and/or games for health). In this regard, the major aim is to educate patients on healthier lifestyle habits (healthy diet and physical activity domains) and to facilitate the process of illness acceptance after a diagnosis of GDM.

Finally, our review shows some noteworthy points:

1. the positive effect of a telemedicine intervention on both diabetes-related medical outcomes and general health perception, energy/vitality, and mental health;

2. a significant improvement in QoL of women with GDM attending an educational program. In the future, these considerations should be taken into account for the management of diabetes during pregnancy.

\section{Pregnancy weight-gain guidance}

Like it or not, eating for two isn't a license to eat twice as much as usual. Use healthy lifestyle habits to manage your pregnancy weight gain, support your baby's health and make it easier to shed the extra pounds after delivery.

There's no one-size-fits-all approach to pregnancy weight gain. Appropriate weight gain for you depends on various factors, including your pre-pregnancy weight and body mass index (BMI). Your health and your baby's health also play a role. If you're carrying twins or other multiples, you'll likely need to gain more weight. Work with your health care provider to determine what's right for you.

- $\quad$ Excessive birth weight

- $\quad$ Early (preterm) birth

- Serious breathing difficulties

- Low blood sugar (hypoglycemia)

- Obesity and type 2 diabetes later in life

- Stillbirth 
The above excerpt covers some key points related to the characteristics, causes, and consequences of gestational diabetes. However, regarding to information of GDM prevention and control via lifestyle intervention, the author would like to share his own T2D research results to illustrate the extra key points. As T2D patient who has suffered many severe complications, his learned knowledge, accumulated experience, and 11 years of dedicated diabetes research work can shed light on prevention and the control of GDM. After all, both GDM and T2D are branches of diabetes where most of their lifestyle requirements and medical treatments are identical.

\section{The author's case of Obesity and Diabetes}

The author was a severe type 2 diabetes patient since 1996. He weighted at $220 \mathrm{lb}$. (100 kg) at that time. By 2010, he still weighed $198 \mathrm{lb}$. with an average daily glucose of $250 \mathrm{mg} / \mathrm{dL}$ (HbA1C at 10\%). During that year, his triglycerides reached to 1161 and albumin-creatinine ratio (ACR) at 116 . He also suffered from five cardiac episodes within a decade. In 2010, three independent physicians warned him regarding his needs of kidney dialysis treatment and his future high risk of dying from his severe diabetic complications.

In 2010, he decided to self-study endocrinology, diabetes and food nutrition. During 2015 and 2016, he developed four prediction models related to diabetes conditions, i.e., weight, postprandial plasma glucose (PPG), fasting plasma glucose (FPG), and $\mathrm{HbA1C}$ (A1C). As a result, from using his developed mathematical metabolism index (MI) model and those four prediction tools, by end of 2016, his weight was reduced from 220 lbs. (100 kg) to $176 \mathrm{lbs}$. (89 kg), waistline from 44 inches $(112 \mathrm{~cm})$ to 33 inches $(84 \mathrm{~cm})$, averaged finger glucose from $250 \mathrm{mg} / \mathrm{dL}$ to $120 \mathrm{mg} / \mathrm{dL}$, and HbA1C from $10 \%$ to $6.5 \%$. One of his major accomplishments is that he no longer takes any diabetes medications since 12/8/2015.

In 2017, he had achieved excellent results on all fronts, especially glucose control. However, during the pre-COVID period of 2018 and 2019, he travelled to approximately 50+ international cities to attend $65+$ medical conferences and made $\sim 120$ oral presentations. This hectic schedule inflicted damage to his diabetes control, through dinning out frequently, postmeal exercise disruption, jet-lag, and along with the overall metabolism impact due to his irregular life patterns through a busy travel schedule; therefore, his glucose control was affected during this two-year period.

By 2020, his weight was further reduced to $165 \mathrm{lbs}$. (BMI 24.4) and his $\mathrm{HbA1C}$ was at $6.2 \%$ without any medications intervention or insulin injection. Actually, during 2020 with the special COVID-19 quarantine lifestyle, not only has he published approximately 400 medical papers in journals, but he has also achieved his best health conditions for the past 26 years. These good results are due to his non-traveling, low-stress, and regular daily life routines. Of course, his rich knowledge, practical experiences, and various developed hightech lifestyle management tools also contribute to his excellent health status since $1 / 19 / 2020$.
On 5/5/2018, he applied a continuous glucose monitoring (CGM) sensor device on his upper arm and checks his glucose measurements every 15 minutes for a total of $~ 96$ times each day. He has maintained the same measurement pattern to present day. Therefore, during the past 11 years, he could study and analyse his collected $\sim 2$ million data regarding his health status, medical conditions, and lifestyle details. He applies his knowledge, models, and tools from math sticks, physics, engineering, and computer science to conduct his medical research work. His medical research work is based on the aims of achieving both "high precision" with "quantitative proof" in the medical findings, not just through linguistic expressions of qualitative words, vague statements, or complex terminologies.

\section{Results}

This section contains the author's summary findings regarding type 2 diabetes prevention and control. However, it should be emphasized that the shorter pregnancy period which is around 10 months with the GDM consequences affect both mother and baby, is unlike his 11-years research period with diabetes complications. Therefore, the correct and necessary medication treatments or insulin injections are required according to the physician's recommendations. During the pregnancy period, lifestyle changes or improvements are extremely necessary and useful for the benefit of mother and baby. At least, a suitable lifestyle management program will add more value to the medical treatments which will not hurt mother or baby.

\section{Weight}

Weight in pregnancy has a close relationship with prepregnancy weight. Besides, weight gain during pregnancy is a common and necessary occurrence. However, excessive weight gain can cause a negative impact on the overall health for both mother and baby, especially with gestational diabetes.

In 1960, the average height of an American woman was 5'4" along with the average weight of $140.2 \mathrm{lbs}$. with a BMI of 24.1 (healthy). However, in 2016, the average height was still 5'4" but the average weight increased to 170.6 lbs. with BMI of 29.3 (overweight and near obese). As a result, the average weight increase of $30.4 \mathrm{lbs}$. has illustrated the unhealthy diet of modern America. The weight increase during pregnancy can induce various chronic diseases, including diabetes along with gestational diabetes, hypertension, and hyperlipidemia, which increases the risk of having many other complications during childbirth including death. Over 15 years ago, a clinical professor at the University of Iowa Hospital shared his experiences with the author regarding the fatality situations related to mothers who were overweight and diabetic. This was before the author heard of the term "gestational diabetes".

Using the average data of "normal and average" American women (non-pregnancy) as an example, the following table lists their weight and corresponding BMI values below. An average height, pre-pregnant woman can plug in her weight to determine her corresponding BMI value. 


\begin{tabular}{|l|l|l|}
\hline Weight (lbs.) & Weight (lbs.) & BMI \\
\hline Y1960 & 140.2 & 24.1 \\
\hline If weight is: & 145 & 24.9 \\
\hline If weight is: & 150 & 25.7 \\
\hline If weight is: & 155 & 26.6 \\
\hline If weight is: & 160 & 27.5 \\
\hline If weight is: & 165 & 28.3 \\
\hline If weight is: & 170 & 29.2 \\
\hline Y2016 & 170.6 & 29.3 \\
\hline
\end{tabular}

In general, for women with different heights and weights, they can utilize the following two formulas to get their BMI values.

The US imperial formula for calculating BMI is expressed as: $\mathrm{BMI}=[($ weight lbs $\times$ 703) $/$ (height inches- squared $)]$

The metric formula is expressed as:

$\mathrm{BMI}=($ weight $\mathrm{kg}) /($ height $\mathrm{m}$-squared $)$

An expectant mother should focus on the "pregnancy weightgain guidelines" set by the Mayo Clinic shown in Figure 1.

\begin{tabular}{|c|c|}
\hline \multicolumn{2}{|c|}{ Pregnancy weight-gain guidelines } \\
\hline \multicolumn{2}{|c|}{ Consider these general guidelines for pregnancy weight gain: } \\
\hline Pre-pregnancy weight & Recommended weight gain \\
\hline $\begin{array}{l}\text { Underweight (BMI under } \\
\text { 18.5) }\end{array}$ & $\begin{array}{l}28 \text { to } 40 \text { lbs. (about } 13 \text { to } 18 \\
\mathrm{~kg} \text { ) }\end{array}$ \\
\hline $\begin{array}{l}\text { Normal weight (BMI } 18.5 \text { to } \\
24.9 \text { ) }\end{array}$ & $\begin{array}{l}25 \text { to } 35 \mathrm{lbs} \text {. (about } 11 \text { to } 16 \\
\mathrm{~kg} \text { ) }\end{array}$ \\
\hline Overweight (BMI 25 to 29.9) & $\begin{array}{l}15 \text { to } 25 \mathrm{lbs} \text {. (about } 7 \text { to } 11 \\
\mathrm{~kg} \text { ) }\end{array}$ \\
\hline Obesity (BMI 30 or more) & 11 to $20 \mathrm{lbs}$. (about 5 to $9 \mathrm{~kg}$ ) \\
\hline \multicolumn{2}{|c|}{$\begin{array}{l}\text { When you're carrying twins or other multiples } \\
\text { Consider these general guidelines for pregnancy weight gain if } \\
\text { you're carrying twins: }\end{array}$} \\
\hline Pre-pregnancy weight & Recommended weight gain \\
\hline $\begin{array}{l}\text { Normal weight (BMI } 18.5 \text { to } \\
24.9 \text { ) }\end{array}$ & $\begin{array}{l}37 \text { to } 54 \mathrm{lbs} \text {. (about } 17 \text { to } 25 \\
\mathrm{~kg} \text { ) }\end{array}$ \\
\hline Overweight (BMI 25 to 29.9) & $\begin{array}{l}31 \text { to } 50 \text { lbs. (about } 14 \text { to } 23 \\
\mathrm{~kg} \text { ) }\end{array}$ \\
\hline Obesity (BMI 30 or more) & $\begin{array}{l}25 \text { to } 42 \text { lbs. (about } 11 \text { to } 19 \\
\mathrm{~kg} \text { ) }\end{array}$ \\
\hline
\end{tabular}

Figure 1: shows pregnancy weight-gain guidelines from Mayo Clinic

She can use the above mentioned "normal weight and BMI range" combined with the "weight-gain allowance" from Mayo Clinic to determine her ideal weight range during pregnancy.

Weight control and Diet

Weight is the most complicated and difficult health issue to deal with because it involves many influential factors with different degree of influences. For a pregnant woman, not only is she concerned about food quantity but also maintaining the necessary nutritional balance.

The author will use his own data record and research results to demonstrate the importance of food and meal quantity, i.e., food portion percentage of "normal portion" on body weight. The term of "normal portion" is defined as a "food quantity baseline" during a period of normal intake amount of food and meals. His food portion during 2012 is used as the $100 \%$ food portion baseline. This was two years after his initial warning from his physician's chronic diseases complication's death threat. He then started to self-study both internal medicine and food nutrition.

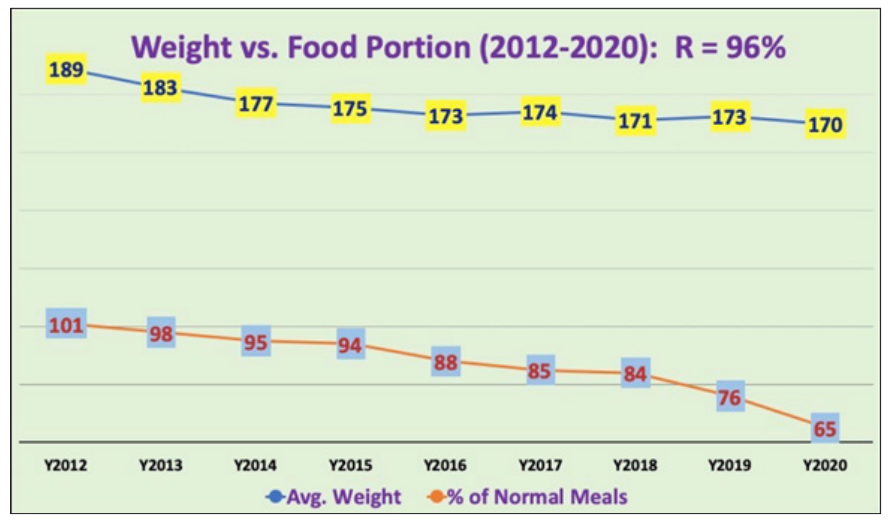

Figure 2: Weight is controlled by food portion \%

Figure 2 reveals the close relationship with the extremely high correlation coefficient of $96 \%$, between body weight and food portion percentage over the 9-year period from 2012 to 2020. His weight decreased from $189 \mathrm{lbs}$. in 2012 to $170 \mathrm{lbs}$. in 2020 while his food portion percentage dropped from $101 \%$ to $65 \%$. In other words, after cutting off 1/3 of his normal meal portion, he reduced his weight by $19 \mathrm{lbs}$. or $10 \%$ of his original weight of 189 lbs. in 2012. From the data, you can see how difficult it was to reduce $10 \%$ of his weight, because it required trimming $35 \%$ off his total food quantity.

However, during the pregnancy period, the mother must eat a healthy and nutritionally well-balanced food. She must choose a diet with plenty of fresh vegetables and some fruits. The best choices are fresh, frozen, and canned vegetables along with vegetable juices without added sodium, fat, or sugar. Good vegetable examples are asparagus, broccoli, cabbage, carrots, cauliflower, celery, eggplant, green leafy vegetables, bell peppers, snap peas, and tomatoes. Tomato is a great vegetable, but the author treats it as one of his daily "fruit" similar to berries. He feels that "one tomato a day, keeps the doctors away". Corn is too starchy and sweet (one ear of corn contains $\sim 30$ grams of carbs/sugar). A serving of vegetables consists of $1 / 2$ cup of cooked vegetables, $1 / 2$ cup of vegetable juice, or 1 cup of raw vegetables. A woman with GDM can consume $\sim 2$ cups of cooked vegetables per meal or $\sim 4$ cups of raw vegetables. 
Whole grains food are also important because the entire grain includes the bran, germ, and endosperm (starchy part). Look for cereals and grains with the first ingredient of whole grain such as bulgur (cracked wheat), whole wheat flour, whole oats/ oatmeal, whole grain corn/corn meal, brown rice, or whole rye. Try to include dried beans, legumes, peas, and lentils into several meals per week. They are a great source of protein and are loaded with fiber, vitamins and minerals. A GDM or diabetes patient should always avoid eat starchy foods that are processed, such as white rice, white flour, spaghetti, noodles, pasta, white toast, potato chips, etc.

Fresh fruits without added sugars are necessary for nutritional balance. Common fruits include apples, blackberries, blueberries, cantaloupe, dates, figs, grapes, oranges, pears, and strawberries. You should avoid sweet fruits, such as bananas, pineapples, and oranges. All kinds of berries are good for glucose control. You should control the amount of other sweet fruits to avoid having hyperglycemia. The author only eats $1 / 3$ of a banana or $1 / 2$ an orange each time at three hours after his previous meal and two hours before his next meal. For example, his lunch is usually around 12:00 noon and his dinner is around 5:00 pm, and he eats 1/3 of a banana or 1/2 of an orange at 3:00 pm. If possible, avoid eating any sweet food or high-sugared snacks because the normal food supply including vegetables already contain varying amounts of sugar.

For a GDM patient's nutritional balance, you should choose healthier options for dairy and meat, including low-fat or non-fat dairy products for milk and cheese, lean meats that are lower in saturated fat and calories. You may be surprised to find out that most of processed dairy foods contain higher carbohydrates and sugars amount than you need. Fish, seafood, poultry without skin, eggs, and choice grades of meat with trimmed-off fat are good for GDM patients. Again, during pregnancy, a GDM patient should avoid sweet or sugar-added food and snacks, if possible.

\section{Most importantly as shown in Figure 2, be sure to watch your food portion size}

\section{Weight and FPG}

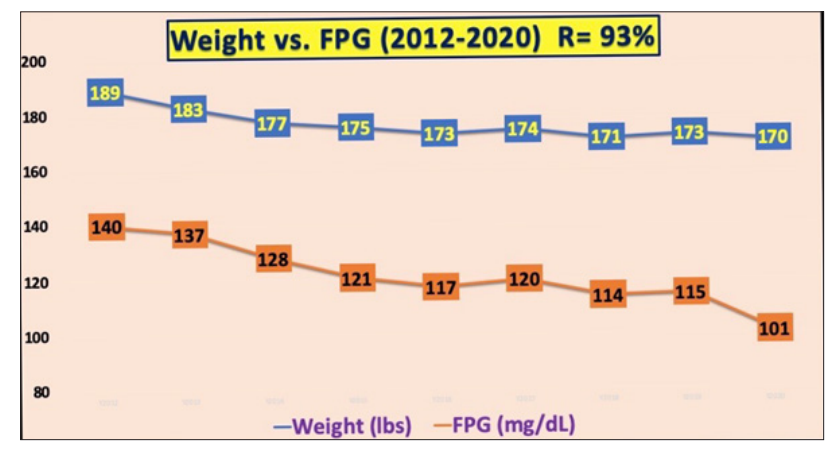

Figure 3: FPG is controlled by weight

Figure 3 indicates another close relationship between weight and FPG indicated by their high correlation coefficient of $\mathbf{9 3 \%}$. During the same 9-year period from 2012 to 2020, when he reduced his weight by $19 \mathrm{lbs}$. (10\% reduction) from 189 lbs. in 2012 to 170 lbs. in 2020, his corresponding FPG was reduced by $39 \mathrm{mg} / \mathrm{dL}$ ( $28 \%$ reduction) from $140 \mathrm{mg} / \mathrm{dL}$ in 2012 to $101 \mathrm{mg} / \mathrm{dL}$ in 2020. In 2015, it took him 9 months to discover and prove the tight correlation between weight and FPG. FPG has about 5 influential factors with weight alone contributing about $80-85 \%$ of FPG formation. Besides, for a T2D or GDM patient, your average FPG value is a good indicator of your current health state for pancreatic beta cells since FPG has no "direct" influences from food and exercise, except for internal hormones, including insulin. Food quantity has a direct impact on weight, which is a direct link to your FPG.

\section{$P P G$ and Carbs intake \& exercise}

According to his research findings, there are 19 influential factors for PPG formation. Among them, carbs/sugar intake amount and "post-meal" exercise occupy around $80 \%$ of the total PPG influences. There are many types of exercise a person can choose. An elderly person and a young pregnant mother are on the extreme ends of the age scale, but the author has identified an easy and high-quality form of exercise for all people which is "walking". Walking provides a positive exercise effect on all parts of the body, including internal organs, and easy to do under most circumstances. He has also identified that "post-meal" walking is extremely effective on bringing down the elevated PPG level within 30 to 60 minutes after consuming meals.

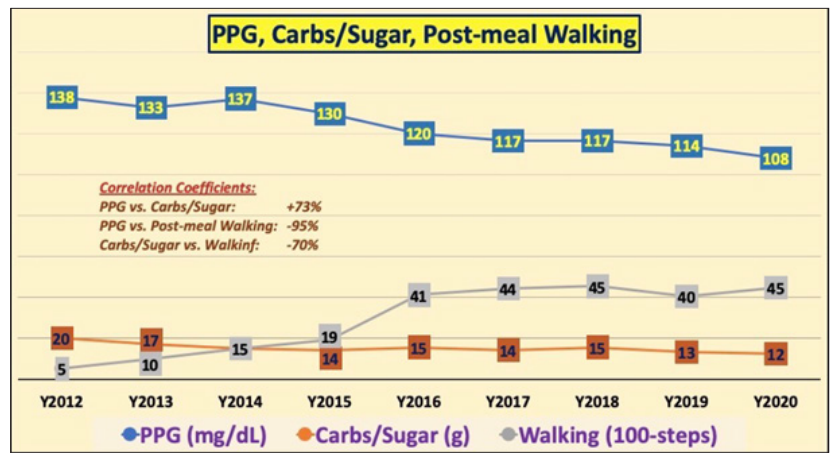

Figure 4: PPG is controlled by both carbs/sugar intake amount (diet) and post-meal walking steps (exercise)

Figure 4 signifies the close relationships between PPG and Carbs intake (with a correlation coefficient of $+73 \%$ ) and between PPG and post-meal walking (with a correlation coefficient of $-70 \%)$.

Even between the carbs intake amount and post-meal walking, they have a high $-70 \%$ correlation between them, but the high negative correlation is caused by the author's learned knowledge and conscientious effort on glucose control.

Regarding the correlation coefficient, a "minus" sign means their trends are in the opposite directions, e.g., the more you exercise, the lower your PPG will be. On the other hand, a "positive" sign means their trends are in the same directions, e.g., the more carbs you eat, the higher your PPG will be. By the way, any correlation coefficient greater than $50 \%$ is considered 
having a "strong" correlation between two variables.

\section{HbA1C and Glucoses (FPG \& PPG)}

The daily average glucose and daily mathematical $\mathrm{HbA1C}$ values are contributed by $\sim 25-30 \%$ from the average FPG and $\sim 70-75 \%$ from the average PPG. The lab-tested HbA1C value is determined by the average glucose situations within the past 3-4 months since the average life span of red blood cells is approximately 3 to 4 months. The life span of liver cells are around 1 year, while pancreatic beta cells live for a long time. Similarly, once beta cells are damaged, it would take an equal amount of time to be repaired, i.e., an extremely slow rate of self-repairing for the pancreatic beta cells. This also explains why a GDM patient is likely to have gestational diabetes for future pregnancies and getting T2D post-pregnancy. All of these situations involve "insulin resistance" from the pancreatic beta cells functionalities and insulin qualities.

The author's lab-tested $\mathrm{HbA1C}$ data for the past 20 years are displayed in Figure 5. As he mentioned in his health history section, he has a history of T2D for 26 years. From Figure 5 , it is obvious that his $\mathrm{HbA1C}$ fluctuated between 2000 and 2010 which indicates his glucose levels were out of control. In 2010, his A1C reached to the peak value of $10 \%$ after he suffered from five cardiac episodes along with having kidney complications. After 2010, he started to self-study and research on both endocrinology and food nutrition. During 2010-2014, his average $\mathrm{A} 1 \mathrm{C}$ was around $6.6-7.3 \%$. He spent the entire year of 2014 to develop a mathematical metabolism index (MI) model in order to understand the complex interactions between 4 medical conditions categories and 6 lifestyle details categories. During 2015 to 2017, he further developed 4 prediction models for weight, FPG, PPG, and HbA1C. As a result, his average $\mathrm{HbA} 1 \mathrm{C}$ dropped to a constant level around $6.5 \%$ without medication intervention. He has continued his glucose control via a refined lifestyle improvements based on his own research findings, where he finally reached an $\mathrm{A} 1 \mathrm{C}$ of $6.1 \%$ in early 2021 .

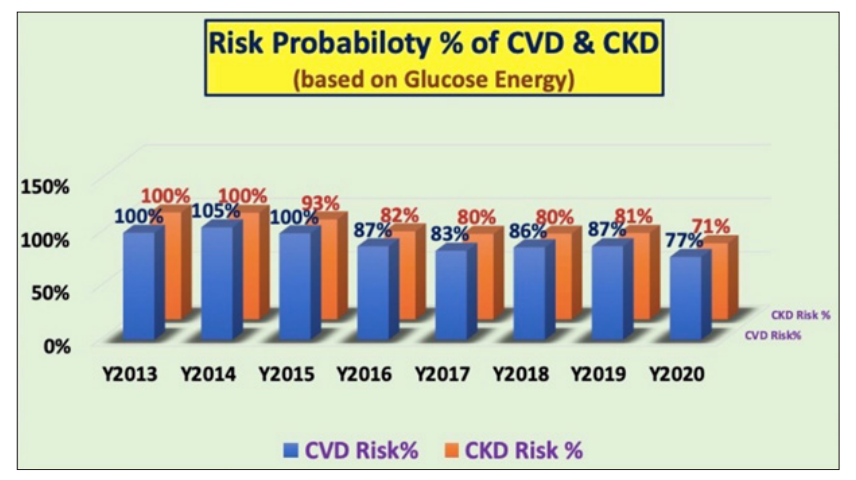

Figure 5: $\mathrm{HbA1C}$ is controlled by both PPG $(\sim 75 \%)$ and FPG $(\sim 25 \%)$

He provides his detailed history of HbA1C improvement record to GDM patients as a reference and proof of having an effective diabetes control via lifestyle management. Although GDM may be a possible symptom of diabetes during the second half of pregnancy, we also know that GDM may be a sign for a continuous long struggle with having diabetes for the rest of her life. Even though gestational diabetes is a serious condition, there is a scientific-based evidence to achieve the goal of controlling this beast and living with it.

\section{Diabetes and its Complications}

The author presents his risk probabilities of having a diabetes complications, such as cardiovascular disease (CVD), stroke, and chronic kidney disease (CKD). This does not mean that a GDM patient would end up having severe complications like CVD and CKD during her short 10-month pregnancy; however, it highlights the interconnections between diabetes and its possible life-threatening complications in the future.

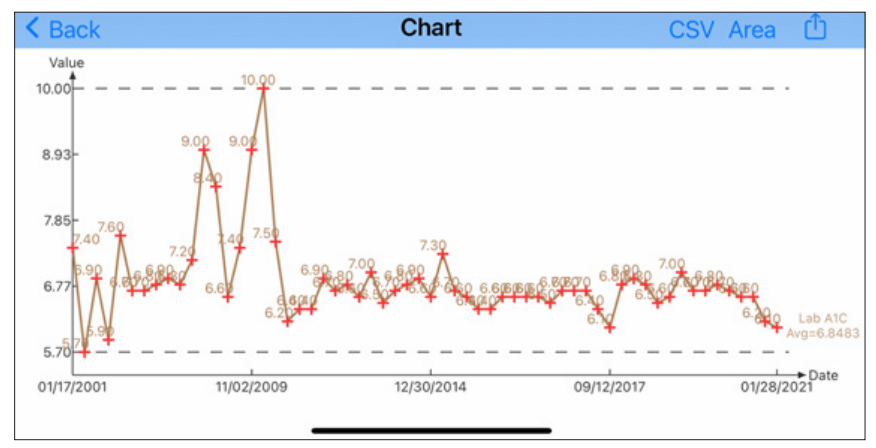

Figure 6: Complications such as CVD, stroke, or CKD are controlled by glucose, blood pressure, \& lipid which are further controlled by lifestyle management details

Figure 6 validates his risk probabilities of having a CVD, stroke, or CKD during the 8-year period from 2013 to 2020. In this diagram, he has normalized his risk percentage using his 2013 risk value as the baseline. It is evident that his CVD/ Stroke risk has reduced from $100 \%$ in 2013 to $77 \%$ in 2020 , and his CKD risk has decreased from $100 \%$ in 2013 to $71 \%$ in 2020. His risk reduction trend and pattern of having chronic diseases induced complications are remarkably similar to his reduction trends and patterns of his weight and glucose during the same period. If you are diagnosed with gestational diabetes, this observation has proven the importance of controlling your weight and glucose at the same time by focusing on the root causes to prevent other complications down the road.

\section{Conclusions}

Although his research specialties are in the areas of endocrinology, diabetes, and lifestyle, after 11 years of dedicated medical research with additional self-studying on GDM, he discovered that approximately $90 \%$ of his diabetes research findings are applicable to this special group.

In summary, three areas with special emphasis are highlighted for GDM patients.

The first and major area is food nutrition in a lifestyle management program. Due to the concerns of hyperglycemia, the author eats high-quality protein, a lot of fish and vegetables, particularly green leafy vegetables, portion-controlled shellfish and fruits, along with avoidance of red meat and sweetened food. However, for a GDM patient, he highly recommends 
them to follow a "nutritional balanced" meal plan, avoiding high-carbs, sugar-based, and processed food in general to prevent unhealthy chemical additives. The fetus in utero needs good nutrition supplied by its mother; therefore, the mother must eat nutritional balanced meals that meet nutritional requirements and glucose concerns.

The second area is specific weight-gain management. The author focuses on the food portion percentage with a strong willpower to resist his food cravings plus persistence each day, so that he can achieve his weight reduction target. However, for a GDM patient, she must constantly monitor the amount of weight gained according to the Mayo Clinic's "weightgain guidelines". Maintenance in this area is important for the benefit of the baby's development and mother's health during pregnancy. However, both of his food portion percentage and persistent weight control are good examples to follow and useful for a GDM patient.

The third area is medication treatment for gestational diabetes. Although the author ceased taking medications in controlling his diabetes symptoms, he decided to implement a rather stringent lifestyle management program in order to control or even reverse his diabetes conditions at the root-cause level. Nevertheless, it is a difficult route that takes a longer time span to be able to see significant improvements. Since the pregnancy period is relatively shorter, less than 10 months, a gestational diabetes patient must follow her physician's advice to pursue all the necessary medication treatments or insulin injections.

The information in particular the high correlation coefficients between any two variables, from Figures 2 through 5, provide useful knowledge for a GDM patient to learn in order to control her glucose levels during pregnancy. The most important goal is the safe delivery of a healthy new born.

\section{References}

1. Gerald C Hsu, eclaireMD Foundation, USA, "Biomedical research using GH-Method: math-physical medicine, version 3 (No. 386)".

2. Gerald C Hsu, eclaireMD Foundation, USA, "From biochemical medicine to math-physical medicine in controlling type 2 diabetes and its complications (No. 387)".

3. Gerald CHsu, eclaireMD Foundation, USA, "Methodology of medical research: Using big data analytics, optical physics, artificial intelligence, signal processing, wave theory, energy theory and transforming certain key biomarkers from time domain to frequency domain with spatial analysis to investigate organ impact by relative energy associated with various medical conditions (No. 397), 2/3-6/2021

4. American Diabetes Association https://www.diabetes. org/diabetes/gestational-diabetes Gestational Diabetes, Treatment \& Perspective.

5. American Diabetes Association, Gestational Diabetes Mellitus, Diabetes Care 2003 Jan; 26: s103-s105.

6. American Diabetes Association, Management of
Diabetes in Pregnancy: Standards of Medical Care in Diabetes-2020, Diabetes Care 2020 Jan; 43: S183-S192.

7. Mayo Clinic, Healthy Lifestyle, Pregnancy week by week, Pregnancy weight gain: What's healthy? From promoting your baby's development to paving the way for postpregnancy weight loss, here's why pregnancy weight gain matters, By Mayo Clinic Staff.

8. Diabetes and metabolism journal (dmj), New Diagnostic Criteria for Gestational Diabetes Mellitus and Pregnancy Outcomes in Korea, Kyu Yeon Hur, Division of Endocrinology and Metabolism, Department of Medicine, Samsung Medical Center, Sungkyunkwan University School of Medicine, Seoul, Korea., Department of Health Sciences and Technology, Samsung Advanced Institute for Health Sciences \& Technology, Sungkyunkwan University, Seoul, Korea.

9. Screening, Diagnosis, and Management of Gestational Diabetes Mellitus, ANDREW GARRISON, MD, Central City Community Health Center and the University of Utah, Salt Lake City, Utah, American Family Physician 91: 460-467.

10. UCSF Health, Gestational Diabetes.

11. Daniela Marchetti, Danilo Carrozzino, Federica Fraticelli, Mario Fulcheri, Ester Vitacolonna (2017) "Quality of Life in Women with Gestational Diabetes Mellitus: A Systematic Review", Journal of Diabetes Research 12 pages.

12. Gerald C Hsu, eclaireMD Foundation, USA, "An experimental study on self-repair and recovery of pancreatic beta cells via carbs/sugar intake increase and associated postprandial plasma glucose variation using linear elastic glucose theory and GH-Method: mathphysical medicine (No. 396)".

13. Gerald C Hsu, eclaireMD Foundation, USA, "A neuroscience model between the brain and liver regarding glucose secretion in early morning using GH-Method: math-physical medicine (No. 394)".

Copyright: (C2021 Gerald C Hsu. This is an open-access article distributed under the terms of the Creative Commons Attribution License, which permits unrestricted use, distribution, and reproduction in anymedium, provided the original author and source are credited. 